\title{
Pharmacogenomic alerts: Developing guidance for use by healthcare professionals
}

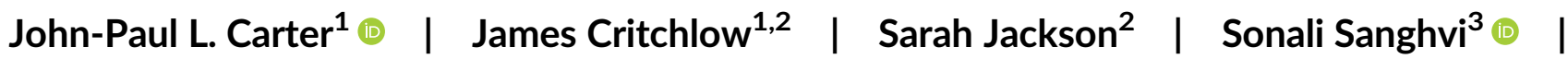 \\ Helene Feger $^{2}$ | Afzal Chaudhry ${ }^{2,4}$ | Lorraine Foley ${ }^{2}$ | Reecha Sofat ${ }^{1}$ (1)
}

${ }^{1}$ Centre for Clinical Pharmacology and Therapeutics, Institute of Health Informatics, University College London, UK

${ }^{2}$ Professional Record Standards Body, London, UK

${ }^{3}$ Genomics Unit, NHS England and NHS Improvement, England

${ }^{4}$ Hospital \& Renal Medicine, Cambridge University Hospitals NHS Foundation Trust, UK

\section{Correspondence}

Reecha Sofat, Professor of Clinical Pharmacology and Therapeutics, Centre for Clinical Pharmacology and Therapeutics, University College London, UK.

Email: r.sofat@ucl.ac.uk

Funding information

NHS England; NIHR Cambridge Biomedical Research Centre, Grant/Award Number: BRC1215-20014; NIHR UCLH Biomedical Centre
Aims: For diseases with a genetic cause, genomics can deliver improved diagnostics and facilitate access to targeted treatments. Drug pharmacodynamics and pharmacokinetics are often dependent on genetic variation underlying these processes. As pharmacogenomics comes of age, it may be the first way in which genomics is utilised at a population level. Still required is guidance and standards of how genomic information can be communicated within the health record, and how clinicians should be alerted to variation impacting the use of medicines.

Methods: The Professional Record Standards Body commissioned by NHS England developed guidance on using pharmacogenomics information in clinical practice. We conducted research with those implementing pharmacogenomics in England and internationally to produce guidance and recommendations for a systems-based approach.

Results: A consensus viewpoint is that systems need to be in place to ensure the safe provision of pharmacogenomics information that is curated, actionable and up-todate. Standards should be established with respect to notification and information exchange, which could impact new or existing prescribing and these must be in keeping with routine practice. Alerting systems should contribute to safer practices.

Conclusion: Ensuring pharmacogenetics information is available to make safer use of medicines will require a major effort, of which this guidance is a beginning. Standards are required to ensure useful genomic information within the health record can be communicated to clinicians in the right format and at the right times to be actioned successfully. A multidisciplinary group of stakeholders must be engaged in developing pharmacogenomic standards to support the most appropriate prescribing.

KEYWORDS

alerts, electronic health records, genomics, pharmacogenomics 


\section{1 | INTRODUCTION}

Pharmacogenomics has the potential to facilitate precision in drug dosing, improve targeting of therapies for diseases, and reduce incidence of adverse drug reactions (ADRs). ${ }^{1}$ However, integration into clinical practice has been limited by the availability of widespread genomic or panel-based testing, and system-wide challenges to enable test results to be available in the electronic health record (EHR) and to be actioned appropriately. ${ }^{2}$ Clarity around clinical utility and cost-effectiveness is also required. Genetic testing by the National Health Service (NHS) has been employed since the 1960s, and the case for whole genome sequencing (WGS), stimulated by the 100000 genomes project led by Genomics England, has made widespread genomics in the NHS a reality. Implementation of genomic testing in clinical pathways is now being facilitated by the Genomics Medicines Service through regional Genomic Medicines Service Alliances (GMSA) with testing delivered by the Genomic Laboratory Hubs (GLH). ${ }^{3}$

Within this context, pharmacogenomics presents itself as an opportunity which could have a population-wide impact as a large proportion of the population either do or will take medicines with possible actionable drug-gene associations (DGAs) during their life course. DGAs occur where the presence of specific genetic variants affects the pharmacodynamics or pharmacokinetics of a drug or class of drugs. For certain genotypes, a DGA may increase the risk of serious ADRs. For example, prescription of the anti-retroviral drug abacavir can cause a life-threatening hypersensitivity syndrome in individuals with the HLAB*5701 allele. $^{4}$ Retrospective UK-based analyses demonstrate that eight out of ten individuals in primary care were exposed to at least one drug whose prescription may have been altered as a result of genomic testing over a 20 -year period. ${ }^{5}$ This represented $16 \%$ of all drugs prescribed. If identified prospectively, this could have the potential to reduce the overall rate of ADRs, many of which have a genomic basis. Together, all ADRs lead to $\sim 6.5 \%$ of hospital admissions in the UK at a (inflation-adjusted) cost approaching $£ 700$ million in $2021{ }^{6}$

To effect this change, however, pharmacogenomic information may need to be actioned in different healthcare settings by different healthcare professionals with different levels of resources. This may or may not include access to electronic prescribing systems and healthcare records, the implementation of which is heterogeneous with varying levels of interoperability. For example, in the NHS such systems are currently more advanced in primary care, with growing use in secondary care and a drive to enhance information sharing between settings and to provide patients with direct access to their personal health record. Although the implementation of electronic sharing of pharmacogenomic information may not be universally possible without electronic systems, the recommendations in this paper-i.e., what information is needed, when and by whom-still apply to settings without EHR provisions.

Before widespread implementation and adoption, certain issues need to be addressed. First is the upskilling of health professionals as pharmacogenomics is a specialised area that often remains beyond the scope of formal clinical training; second, a mechanism of codifying genomics data and allowing the flow of test results from laboratory to the health care record in a system-agnostic manner and in line with

\section{What is already known about this subject}

- Most individuals carry undiagnosed actionable pharmacogenomic variants with specific drug gene associations (DGAs), and identifying these before prescribing a relevant medicine may reduce avoidable adverse drug reactions.

- Both in the British National Health Service (NHS) and internationally, the routine integration of pharmacogenomics into real-world clinical practice is in its infancy, with the majority of implementation at the pilot/prototype stage.

- Barriers to adoption include a lack of clinician/patient familiarity with the subject, immature clinical decision support systems, and limited national guidance and lack of initiatives such as national programmes for preemptive pharmacogenomic testing.

\section{What this study adds}

- This study highlights and outlines the development of system-wide guidance in the United Kingdom for the sharing of pharmacogenomic information with prescribers, health professionals and patients.

- This study, which was based on an evidence review and wide multidisciplinary consultation, is consistent with and supports and adds to the findings of a recently published US review and guidance for pharmacogenomics clinical decision support by Wake et al. ${ }^{19}$

- This study provides a foundation for further work including the future development and piloting of a national pharmacogenomics information record standard to support interoperability of pharmacogenomics information systems.

national and international interoperability standards is required; and third, and the focus of this review, is how to best alert healthcare providers to enable them to action a pharmacogenomic result.

\subsection{Aim and scope of the project}

The project aimed to provide guidance on how genetic information that could affect prescribing decisions can be communicated effectively and actioned to improve prescribing choices. Through the course of the project, key challenges to implementation of pharmacogenomic alerts as a systems approach were identified and 
recommendations were made. The scope of the project was from a UK-based NHS view, where pharmacogenomic testing and alerting is not part of routine clinical practice.

\section{2 | METHODS}

\subsection{Project overview}

A project board was convened by the Professional Record Standards Body (PRSB) in collaboration with NHS England and NHS Improvement (NHSE/I) comprising individuals with experience of health records, prescribing and pharmacogenomics across a range of specialties, healthcare backgrounds from both primary and secondary care as well as patients. The project board was chaired by the CEO of the PRSB and the clinical lead of the project was a doctor with expertise in prescribing and genomics. The board was responsible for ensuring the project team met key deliverables outlined in the overview (Figure S1 in the Supporting Information), meeting regularly from September 2019 to March 2020 and culminating in presentation of the findings to NHSE and a report in June 2020.

\subsection{Field synopsis}

A field synopsis was carried out with the aim of understanding the scale and scope of pharmacogenomic alerts and how they had been deployed in health record systems to date. The search was abbreviated, necessarily limited by time and budget constraints, but also by the fact that the literature on pharmacogenomic alerts in healthcare compared to electronic healthcare records remains in a pilot or prototype stage. Details of the search terms are given in the Supporting Information. The field synopsis was key to informing a preliminary set of discussion points initially for a focus group meeting. It also allowed identification of research and clinical groups working on pharmacogenomic alerting systems for further consultation, and provided peer review evidence that could be incorporated into the draft guidance, which was iteratively reviewed through consultation and focus groups with key stakeholders (Figure S2 in the Supporting Information).

\section{$2.3 \quad$ Focus groups}

Focus groups comprising key stakeholders, including but not limited to patients, clinicians, informaticians and pharmacists from a range of specialities and spanning primary and secondary care, were convened to explore the scope of pharmacogenomic alerts with a focus on improving safety and care of individuals. Two focus groups were held: the first aimed to explore the scope of guidance for pharmacogenomic alerts within the context of the NHS and the second was to review and contribute to the draft guidance, ensuring it was practicable, understandable and fit for purpose. Views were sought on the draft guidance and were illustrated by several clinical scenarios. Timelines of these and the overall framework of the project are outlined in Figures S1 and S2 in the Supporting Information.

\section{4 | International expert interviews}

The field synopsis was used to identify key groups internationally that have experience of deployment of pharmacogenomic test alerts within clinical practice and with a mixture of pre- and post-test alerting systems as well as any clinical decision support systems that were in place for pharmacogenomics. This was followed by conducting semi-structured interviews with key international groups (Supporting Information) and summarised as feedback for both focus groups and the consultation survey. This included stakeholders from the United States $(n=6)$, Canada $(n=1)$, Holland $(n=1)$, Italy $(n=1)$ and the UK $(n=4)$. Stakeholder roles included clinicians, professors and researchers in adult and paediatric medicine, pharmacy, pharmacogenomics and biomedical informatics, as well as independent consultants in informatics. All international stakeholders were involved in researching, directing, coordinating, researching or piloting pharmacogenomic services in university or research hospitals.

\section{5 | Consultation survey}

Consultation calls were held with patient representatives and UKbased professionals, including representations from key learned societies to further inform the draft guidance. The discussions included feedback on the preliminary findings of the field synopsis and international surveys to help inform development of the draft guidance.

\section{6 | Twitter chat with patient representatives}

To gather additional patient perspectives, the PRSB organised a Twitter chat with patient representatives, entitled 'What does your clinician need to know about your genes?'.

\subsection{Draft and final guidance and report}

Draft guidance was produced for review that, following focus groups and consultation, was revised to produce final guidance in the form of a report and key recommendations.

\section{3 | RESULTS}

\subsection{Field synopsis professional and patient consultations}

The abbreviated field synopsis was carried out to assess the scope and scale of pharmacogenomic alerts internationally and how they are 
utilised as well as inform themes for the focus groups and consultation. Four key themes emerged from this review. First, what information was being alerted; second, how the information was being presented; third, how the information was available through the lifecycle of a prescription (Figure 1); and fourth, how this process could evolve iteratively. These and other themes enabled an initial focus group and international consultation and the formation of a draft guideline. This was then reviewed and a final guideline agreed upon.

\section{2 $\quad$ Current state of implementation and classification of pharmacogenomic alerts}

No national UK-based implementation strategies were identified, rather strategies that were centre-based pilot and/or prototype were identified. Through both field synopsis and direct consultation, it was identified that the buy-in from prescribers and rates of clinical adoption were highest when recommendations were simple and

(A)

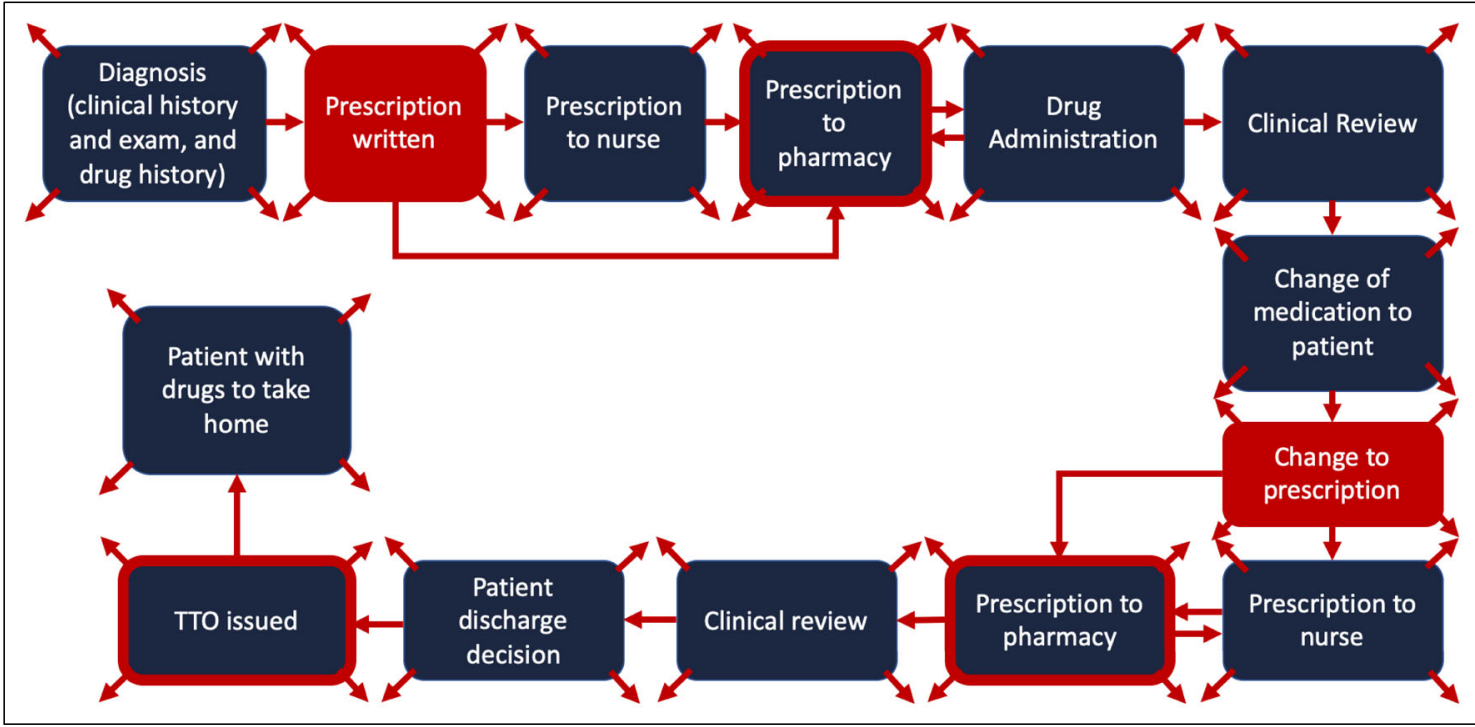

(B)

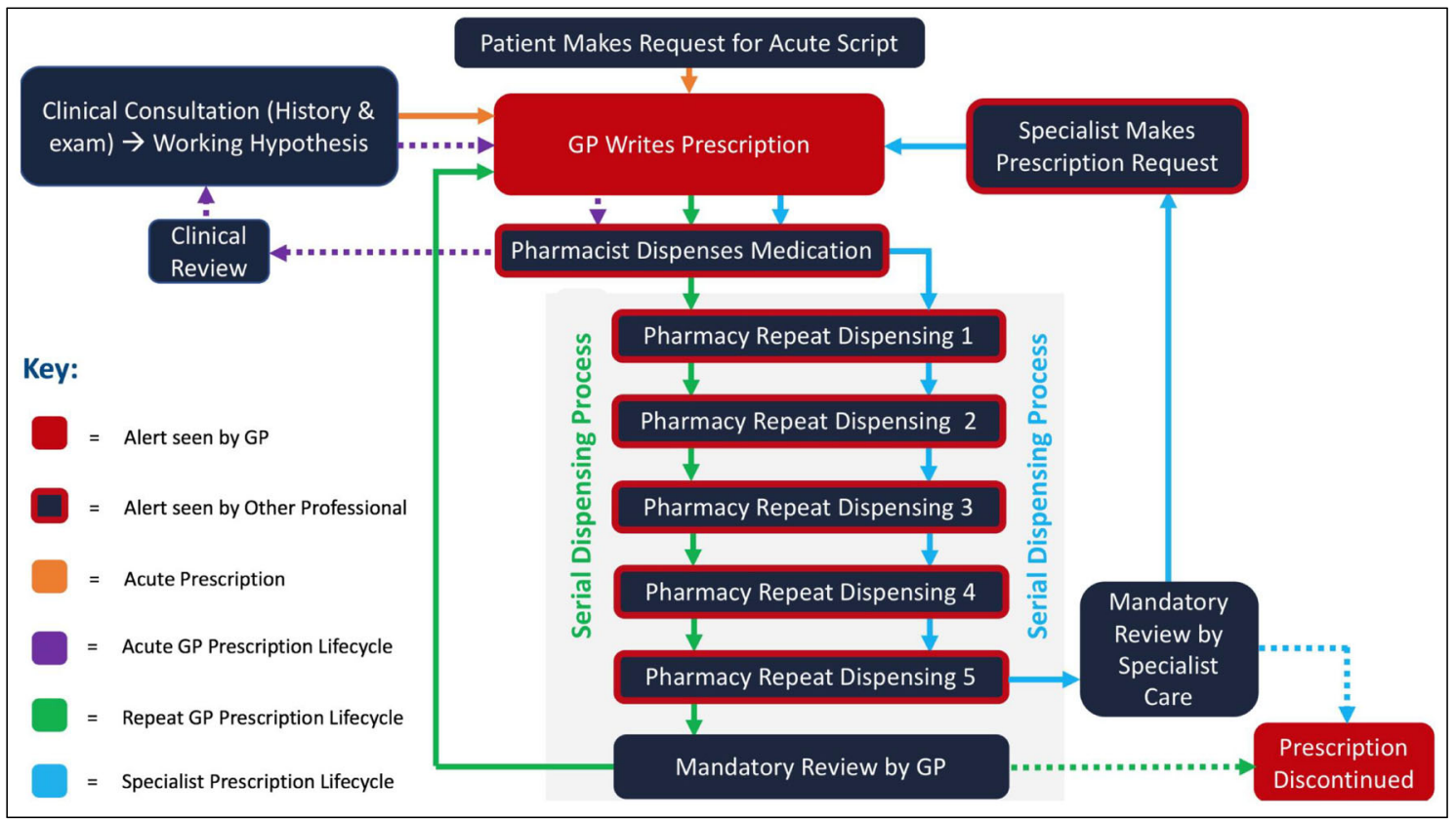

FIGURE 1 The life cycle of a prescription, highlighting key points, which, if pharmacogenomic information were available and could be actioned, may alter the course of prescribing/dispensing of a medicine. Figure $1 \mathrm{~A}$ indicates the possible lifecycle within a secondary care setting and Figure $1 \mathrm{~B}$ in the primary care setting. The arrows emanating from each step indicate known and unknown factors that could impact on that part of the lifecycle of a prescription. This is a general depiction, used for illustrative purposes during the consultation, of how errors might creep into the use of medicines, and pharmacogenomics is one element of this 
prescriptive. Appropriate and useful alerting was found to include information that was clear and unambiguous and where the end-user could evaluate the alert at a glance. Alerts, which can be pre-test or post-test, were triggered most commonly at the time a prescription is written, although these can also be triggered at other times. It was noted that there was no robust evidence (e.g., randomised controlled trials) that demonstrates clear safety advantages of particular types of alert-for example, electronic alerts such as active, passive, hard or soft stops or physical (e.g., patient-held alert cards or bracelets). The consensus view was that no firm recommendations on the type of alert could be made and in fact this may vary depending on the DGA and the context in which the alert is to be displayed.

A classification of alerts emerged within this theme (Figure 2). Post-test alerts are triggered when an individual has a pharmacogenomic test already recorded in the system. These are available within the system when required to be actioned and are a result of sequencing or panel-based testing that has already occurred. Pre-test alerts are triggered where an individual has not yet been tested. The choice of a drug within the EHR may trigger the prescriber to request the appropriate test. Further subcategories of alert were identified. These included active alerts, which are interruptive and should be actioned for the end-user to progress further in the workflow; and passive alerts that are notifications as well as informational alerts which appear in the workflow but are optional to review or respond to. There may also be 'passive' links to internally or externally curated guidance or external sources of information.
Where deployed, it was identified that pharmacogenomic alerts are generally, but not always, electronic pop-ups when used within an EHR and use static text to convey information such as the reason for the alert, the relative importance of the alert, a summary of the relevant DGA or phenotype, a recommended course of action and options (e.g. via radio buttons) as well as links to further resources.

As well as electronic alerts, physical alert cards were also described for use in settings which have not introduced electronic systems. ${ }^{7}$ These were highlighted as part of the European U-PGx project, which included a QR code linked to a website providing customised prescribing recommendations for settings with limited or no in-house electronic clinical decision support systems. ${ }^{8}$

It was clear, however, that whilst there is increasing activity and interest around pharmacogenomics, its uses are largely limited to specific clinical specialties within organisations or in pilot or prototype projects. There are no examples of national programmes that have implemented pharmacogenomic testing and alerting. ${ }^{9}$ The reasons for this were explored and are summarised below.

\section{3 | Identification of implementation challenges}

Through the process, key challenges for the implementation were identified. These are summarised in Box 1 and can be categorised in three themes. The first is establishing what the system challenges are in the communication of genetic test results between laboratories and
FIGURE 2 Flow diagram of the classification of alerts. Pharmacogenomic alerts are generally, but not always, electronic and follow one of two systems outlined here. Firstly, the pre-test alert where pharmacogenomic information is unknown although this may be requested because of a prescribing choice. Secondly, the post-test alert, where pharmacogenomic information is already available as part of sequencing or panel-based investigations. Both types of alerts can be further subdivided into active and passive. Active alerts are interruptive and should be actioned for the end-user to progress further in the workflow. Passive alerts are informational and appear in the workflow but are optional to view or respond to. There may also be passive links to internally curated guidance or external sources of information

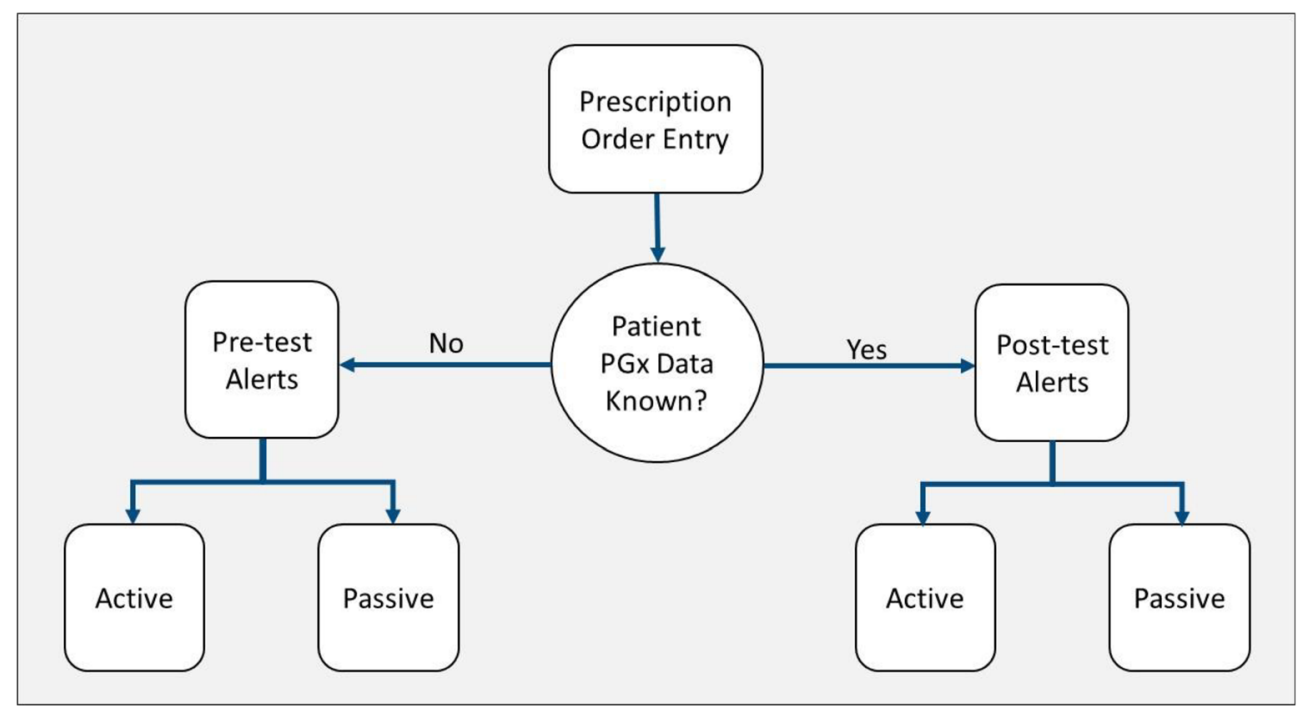




\section{BOX 1 Key implementation challenges of pharmacogenomics identified by the project into a publicly funded system e.g., the NHS. Such challenges could apply to any healthcare setting}

Implementation challenges:

1. Alert fatigue.

2. Clinical systems, data and the need for iterative analysis.

3. Clinical responsibility and oversight.

4. Education and training.

5. Patient-specific considerations.

their entry into patient records. Verbal evidence from national and international experts demonstrated that most systems require a report to be uploaded to an EHR and, in their absence, rely on paper-based reports. The functionality for recording and allowing genomic test information across interoperable systems remains immature although it was noted that there were standards in development e.g., the Fast Healthcare Interoperability Resources (FHIR). These are key to system agnostic implementation and communication of actionable results. Other system challenges include alert fatigue and the need for guidance and oversight of pharmacogenomic clinical decision support systems (CDSS).

The second theme that was identified was patient factors, that is the co-existence of diseases and/or prescription of drugs (e.g., multimorbidity and polypharmacy) which may alter the action of any alert that is delivered. A comprehensive integrated system is desirable and will need to be developed. This could conceivably involve the use of artificial intelligence. The third theme was that of education enabling buy-in for all those involved with alerts from patient to healthcare providers (including those prescribing, dispensing or administering medicines). Education within the training of relevant healthcare disciplines at all levels is key. This will allow evidencebased implementation and adequate communication with patients. These challenges are described in more detail below.

\subsection{1 | Alert fatigue}

Alert fatigue was highlighted as a key barrier to successful implementation. This is a desensitisation phenomenon that occurs when electronic CDSS is utilised in live clinical settings where the sheer number of daily alerts and notifications (many of which may have a limited clinical consequence) results in users ignoring or overriding the alert. A retrospective analysis of 296000 prescriber-patient interactions where a CDSS alerted co-prescription of opioids and benzodiazepines was highlighted as an example of what a detrimental impact alert fatigue could have. ${ }^{10}$ Whilst the domain of pharmacogenomics is no different to other areas of patient safety with respect to prescribing, it was noted that this could add another layer that needs to be taken into consideration around the safe and effective use of medicines.

Mitigation strategies of alert fatigue pertaining to pharmacogenomics were also considered. These included sharing only actionable recommendations within the alert itself, triggering alerts for the highest risk DGAs, and suppression of low priority alerts through, for example, a risk stratification process akin to those deployed for drug-drug interactions (DDI), which could then be monitored by routine audits. ${ }^{11}$

Ergonomic alert design could be a mechanism to avoid alert fatigue. Information should be presented in the order that it is needed and the priority of the alert should be clearly illustrated. The format of the alert should provide easy access for additional information as well as the functionality to enable the person alerted to perform necessary actions.

\subsubsection{Clinical systems, data and the need for iterative analysis}

Most polymorphisms that underlie pharmacogenomic testing are germline mutations. ${ }^{12}$ It therefore follows that due to this permanence of an individual's 'pharmacogenomic burden', testing should only need to be conducted once in a lifetime. However, the difference between panel-based testing and whole genome sequencing was considered. Panel-based testing may only cover DGAs for which there is a current evidence base; however, as drug development evolves, these may increase. Discussion supported by the literature reinforced the ethical duty to update genomic information as the evidence base changes and to ensure that these test results are available at the point of care. ${ }^{13}$ The infrastructure that may be required for this was also discussed. Genomic data storage needs to be secure, accessible, enduring and in a format that facilitates re-analysis. International consultations highlighted that these were not in place in local settings let alone the challenges that would be involved for a national system.

Open questions remained about where genomic (including pharmacogenomic data) should be stored within the EHR. Early attempts to store pharmacogenomic data have included those in allergy or problem lists rather than a unique repository, i.e., how laboratory results are currently handled. More novel methods of storage, however, are evolving, including Genomic Archiving and Communication System (GACS) servers to store pharmacogenomic information within an EHR-this model could also serve for genomic information in general. ${ }^{14}$ It was accepted that pharmacogenomic information is a branch of all genomic information, and whilst it could be a useful leader in addressing data and communication of genomic data issues, it is likely that the systems for genomics and pharmacogenomics will converge.

\subsection{3 | Establishing clinical responsibility and oversight in pharmacogenomics, including selection of drug-gene pairs}

Pharmacogenomic testing is currently routinely offered in the NHS for abacavir and fluoropyrimidines within national guidance. In 2020, 
NHSE published a commissioning policy for pharmacogenomic testing of polymorphisms in the DPYD gene to support detection of patients at high risk of toxicity from fluoropyrimidine chemotherapy, such as 5-fluorouracil, capecitabine and tegafur. ${ }^{15}$ Processes are being established to evaluate other pharmacogenomic tests for addition to the National Genomic Test Directory, with national implementation supported by the GMS infrastructure across England. These developments will significantly change the landscape of how any genomic testing, diagnostic as well as pharmacogenomic testing can be deployed nationally. The National Test Directory will have a section of approved and nationally funded pharmacogenomic tests. This may begin to provide a governance framework within which pharmacogenomic testing and communication can be managed, although it is recognised that individual organisations as well as practitioners will also have a responsibility to act on results appropriately.

There was consensus that DGAs that are implemented at scale should be supported by a robust evidence base and incorporation in treatment pathways, including national guidance such as that published by the National Institute for Health and Care Excellence (NICE), should be considered. Implementation in England could be supported by GMSA and NHS medicines optimisation committees at both local and regional levels. The evidence for clinical utility and cost-effectiveness of the test was thought to be as important as the evidence of using the drug, which would ultimately be publicly funded. Like drug evaluations or health technology assessments (HTAs), such a system of review would then enable a judgement based on evidence as well as cost-effectiveness.

Our review and consultation highlighted the limited nature of current guidance around both the evidence base and implementation protocols for pharmacogenomic tests, including those from regulatory bodies such as the European Medicines Agency (EMA) and the Food and Drug Administration (FDA) as well as specialist bodies including the Clinical Pharmacogenomic Implementation Consortium (CPIC) The NHS could therefore be well positioned to delineate the process of evaluation and implementation and be an exemplar of how pharmacogenomics can be implemented as a national programme.

An area that the group was not able to reach consensus on was where clinical accountability lies with respect to who should be notified, interpret or disclose and take action when pharmacogenomic test results were returned. The field synopsis highlighted findings from the PREDICT (Pharmacogenomic Resource for Enhanced Decisions in Care \& Treatment) pharmacogenomics clinical decision support (CDS) programme which assigned responsibility for acting on a test to various health professionals, including the primary care provider, the provider who ordered the test and the provider who may have previously prescribed a drug affected by the DGAs and the specialist treating the medical condition. ${ }^{16}$ Whilst pharmacogenomic tests should not be considered different to other tests carried out within a healthcare system, it does highlight the need for an oversight process that could be matched to existing medicines and results workflows as outlined.

The literature and stakeholder reviews highlighted that while overall implementation of pharmacogenomics is desirable, it does not override the determination of clinical need and/or judgement. Some patient factors may preclude actioning of pharmacogenomics, although it was also noted that implementation may also add to medicines optimisation. Multi-morbidity and polypharmacy were key areas that were identified as both benefiting as well as precluding uses of pharmacogenomics. It was noted that patient-related factors, e.g., accounting for end organ damage when prescribing, would be desirable and its incorporation may enhance pharmacogenomic alerts.

\subsubsection{Education and training}

The importance of the role of education and training was highlighted. Most of those involved in the lifecycle of a prescription (Figure 1) currently have limited exposure to pharmacogenomics (or genomics) during their training. They may not feel comfortable in handling genetic information unrelated to their speciality or their role. Whilst pharmacogenomic alerts could be used as 'just-in-time' guidance, education and training at all levels need to become established, including role-specific designs and competency standards.

\subsection{5 | Patient-specific considerations}

Patients and end-users were involved at all stages of discussion and the consultative process. They highlighted the need to know how gene variants would be identified and how these would have implications for their treatment as well as how this would be actioned. They also reinforced the need for continual updates as the evidence evoIves, which may require ongoing medication reviews. These findings were supported by a meta-analysis which investigated the patient and healthcare provider 'needs and preferences' relating to pharmacogenomic testing with recommendations including: delivery of results by appropriate healthcare professionals who could provide clarification, interpretation and emotional support; adjunctive tools (e.g., written information) for the purposes of consolidation and recall of the information exchanged as well as resources (e.g., videos) with examples and vignettes to increase understanding; prioritising the use of simple language with the avoidance of scientific jargon. ${ }^{17}$

\section{4 | Key recommendations in final guidance and report}

The activities throughout the project converged on key principles associated with alerting for pharmacogenomics. These form the basis of the recommendations which are summarised in Box 2. A summary is presented here.

\subsection{1 | Dual alerting strategy}

Alerts should be used as part of a dual strategy at the prescribing and the dispensing stage. Justification of an action taken by a prescriber should be displayed, where appropriate, to a healthcare professional 


\section{BOX 2 Key recommendations that emerged from the project pertaining to pharmacogenomic alerts}

\section{Key guidance recommendations:}

1. Dual alerting strategy at prescribing and dispensing stages.

2. Only actionable drug-gene associations should be alerted.

3. Implementation of pre-test and post-test pharmacogenomic alerts.

4. Alerts should provide clear, unambiguous, evidence based, and specific best practice recommendations for action.

5. Alerts should be seen by all healthcare professionals with prescribing or dispensing authority involved in a person's care.

6. Implementation of pharmacogenomic clinical decision support should be overseen by a multi-disciplinary oversight committee.

downstream of a prescription, e.g. why a particular dose reduction occurred or an alert was overridden. All healthcare professionals with a duty of care should have access to the pertinent longitudinal information relevant to a person's prescription. Consideration should be given to the fact that community pharmacists supply an increasing number of over-the-counter medications that may need to be alerted independently of a prescription being made.

\subsection{2 | Actionable DGAs}

Clinical decision support should only be in place for actionable DGAs, although the pharmacogenomic test result should still be accessible within the EHR. Alerts should not be used where the pharmacogenomic test result does not imply an actionable variant or where other factors clearly supersede an individual's pharmacogenomics. The display of alerts that cannot be actioned may contribute to unintended clinical consequences such as alert fatigue. Alerts (including physical alert cards) are not required for patients without actionable variants, even in those individuals from a high-risk ethnic group. In some circumstances a DGA not previously considered to be actionable may be reclassified as actionable in the light of new evidence. In such cases the responsible healthcare professional should be alerted if there is likely to be a material impact on a person's care.

\subsection{3 | Pre-test and post-test alerts}

Where pharmacogenomic tests are first available (post-test) they should be actioned or when prescribing a new drug to which the result is relevant. Physical alert cards should only contain information about validated test results that are specific to the cardholder. Where pharmacogenomic tests are not available (pre-test), as evidence evoIves this should trigger a test being requested when the necessary infrastructure is in place to enable this. Prescribers should be made aware where pharmacogenomic data cannot be retrieved by the system, to avoid clinical errors. If a test result has been ordered but the results are not yet available, a prescriber should be notified at the point of prescribing. Most alerts will likely occur at the time a prescription is written, but healthcare professionals should also be alerted when a pharmacogenomic test result first becomes available in the system. If this shows an actionable variant, this should prompt a review of the medications the person is taking.

\subsubsection{Specific and actionable recommendations}

Information that is shared in an alert should be up-to-date and reflect best practice. Information in the alert should provide evidence-based, clear, unambiguous and specific actionable recommendations for the end-user with access to further information as required. Details provided of the variant and/or phenotype should be understandable for healthcare professionals. Information should also be readily explainable to patients and, if asked for, made available to them in a format that they can understand, e.g. plain language summaries.

\subsection{5 | Presentation of alerts to healthcare professionals}

Alerts should be presented in multiple clinical settings and should be seen by all health professionals with prescribing or dispensing authority involved in the patient's care. Patients carrying alert cards or other physical alerts should be encouraged to show this to all prescribers responsible for their care before a prescription is written and to the dispensing pharmacist, at every contact.

\subsection{6 | Consequences and oversight}

Implementation of alerts should consider unintended consequences including but not limited to alert fatigue. Moreover, implementation should be overseen by multidisciplinary oversight committees and consider at the outset expert support in pharmacogenomics. Monitoring and actioning of alerts should be carried out for quality improvement. Alerts should not place an undue burden on prescribers, pharmacists or patients/end-users. Clinical decision support systems must include (but not be limited to) relevant alerts where pharmacogenomic testing for a drug is mandated by existing professional guidance standards. For example, British prescribing guidelines mandate testing for the HLA-B*5701 allele when prescribing abacavir due to the risk of abacavir hypersensitivity syndrome and the HLAB*153 allele when prescribing carbamazepine due to an association 
with Stevens-Johnson syndrome. There is a need for further pharmacogenomic testing standards to be developed for the NHS covering other actionable DGAs. Appropriate specialist input should be sought when considering a decision to stop a treatment already underway on the basis of newly alerted pharmacogenomic information.

The guidance produced as a result ${ }^{18}$ of this exercise provides an essential foundation for the next important step of developing an information standard for pharmacogenomics.

\section{4 | DISCUSSION}

This project was able to achieve consensus on a broad range of issues regarding pharmacogenomics and its implementation specific to how this information flows from bench to bedside in the form of actionable alerts. It was acknowledged that implementation of pharmacogenomic information into clinical practice has the potential to optimise the use of medicines. As genomics comes of age, the way in which this information flows through the system and reaches a point at which it is actionable requires attention. The information can and should be shared at different points in the clinical workflow using methods of clinical decision support appropriate for the setting. This project highlighted that interruptive alerts have been used as the failsafe mechanism when a prescription is likely to result in significant harm to the patient. However, as pharmacogenomics and genomics in general evolve, as do clinical systems, methods need to be developed that ensure that results can be delivered in a system-agnostic manner and proprietary systems and providers adhere to interoperability and open standards. Here professional standards developers such as the PRSB can define standards which can then use existing platforms (e.g., FHIR) to ensure appropriate knowledge of a given standard is communicated to those that need to action it.

A key barrier of alert fatigue was highlighted and novel mechanisms that might mitigate this should be considered. Staff that need to interpret and action pharmacogenomic alerts should be adequately trained. The field of genomics, including but not limited to pharmacogenomics, now needs to be part of the curricula offered to a wide range of medical and allied medical specialties as it becomes commonplace in healthcare. Importantly, as with all clinical results and conditions, patients should be informed and involved at each stage of the lifecycle of a prescription and this should now include pharmacogenomic information.

A few additional points are worthy of discussion which were raised although discussed in less detail and will need to be considered. First, the setting for this work was the NHS, however, these issues will apply to multiple healthcare arenas, including those that are publicly and privately funded. Second, whilst the focus has largely been on computerised systems, the general principles of having up-to-date information available for it to be clinically actioned applies to settings which may not yet have electronic capabilities. Third, whilst there are leading examples of pharmacogenetic testing, it was acknowledged that there is a rich literature around the ethnic variation of polymorphism which may alter drug metabolism. Systems will need to take this into account and it will need to be built into guidance. Finally, the integration of genomics in general into the health record and any implications beyond healthcare raised issues that have been discussed in the literature for some time. This needs to be an ongoing and evolving conversation with citizens.

Our work is consistent with and adds to the findings of a recent US review and guidance for pharmacogenomics clinical decision support outlining the "five rights of CDS" that successful implementation of pharmacogenomics into clinical practice depends upon. ${ }^{19}$ This requires "the right information" (providing actionable recommendations aligned to evidenced-based guidelines); "the right person" (including alerting pharmacogenomic information only to healthcare professionals and patients who want and need to see it, respectively, and in a "patient-friendly-language"), "the right interventional format" (e.g., active versus passive alerts "that are not overly time-consuming for a busy clinician"), "the right channel" (for access to pharmacogenomic information, e.g. an EHR, webpage or portal, or "stand-alone clinical systems"), and interventions at "the right time" (in the workflow). The authors also emphasise the importance of multidisciplinary stakeholder oversight of pharmacogenomics services.

\section{1 | Recommendations for future work}

One of the key recommendations for future work was a pilot around a use-case. Since the time of writing, an urgent commissioning policy on DPYD testing has been published. Efforts for system-wide implementation of this policy may highlight key barriers or gaps in the workflow that need to be addressed as well as research opportunities for pharmacogenomics in clinical practice.

Recommendations for further national development include establishment of shared information and technical standards, including information governance. This should include all genomic information of which pharmacogenomics is one part. Prescribing and a framework of implementation for DGAs, including the evidence by which they should enter the National Test Directory, need to be formalised, particularly working across agencies including NHSE, NICE and Medicines and Healthcare products Regulatory Agency (MHRA) for newer drugs that are developed with companion diagnostics. Education and upskilling of all those involved with prescribing who may need to interpret or action a pharmacogenomic test is key as are communications with patients and end-users. Specifically for alerts, if these are implemented in a computerised system, mitigating alert fatigue is essential.

Further research areas could address specific identified barriers for health system-wide implementation, as well as research around alert fatigue given the different formats of alerts.

\section{5 | CONCLUSIONS}

The rapid advancement and implementation of genomic technologies across the healthcare system highlights the need for genomic data to be incorporated effectively into patient healthcare records. 
As we deepen our understanding of how medicines interact with our genes, we can harness this knowledge to improve patient care through better disease diagnostics and prognostication, avoid harm and gain benefit through targeted prescribing. Effective systems to link genomic data with electronic health records and clinical decision support for prescribing will be key to realising the benefits of pharmacogenomics.

\section{ACKNOWLEDGEMENTS}

A.C. is supported by the NIHR Cambridge Biomedical Research Centre (BRC-1215-20014). R.S. is supported by the NIHR UCLH Biomedical Centre. The PRSB was commissioned to carry out this project for NHS England.

\section{CONTRIBUTORS}

J.-P.L.C. contributed to the study design, research process, reviewed and edited project deliverables, led focus groups and drafted the manuscript. J.C. performed research and drafted key project deliverables and drafted the manuscript. J.-P.L.C. and J.C. contributed equally to this work. S.J. represented the PRSB and provided project management support, drafted project deliverables and reviewed the manuscript. S.S. represented NHS England and reviewed project deliverables and the manuscript. H.F., A.C. and L.F. reviewed project deliverables and the manuscript. R.S. provided academic and clinical leadership, contributed to the study design, led the research and review of project deliverables, and supported drafting and review of the manuscript. Reecha Sofat was the Principal Investigator for this paper.

\section{COMPETING INTERESTS}

The authors have no conflicts of interest to declare.

\section{DATA AVAILABILITY STATEMENT}

Research data are not shared.

\section{ORCID}

John-Paul L. Carter (D) https://orcid.org/0000-0002-2284-3362

Sonali Sanghvi (D) https://orcid.org/0000-0002-1937-5430

Reecha Sofat (D) https://orcid.org/0000-0002-0242-6115

\section{REFERENCES}

1. Pirmohamed M. Pharmacogenetics and pharmacogenomics. Br J Clin Pharmacol. 2001:52(4):345-347. doi:10.1046/j.0306-5251.2001.0 1498.x

2. Al Kawam A, Sen A, Datta A, Dickey N. Understanding the bioinformatics challenges of integrating genomics into healthcare. IEEE J Biomed Health Inform. 2018;22(5):1672-1683. doi:10.1109/JBHI.2017.2778263

3. NHS England. NHS Genomic Medicine Service. [Internet]. 2021 https://www.england.nhs.uk/genomics/nhs-genomic-med-service/. Accessed November 30, 2021

4. Khan DA. Pharmacogenomics and adverse drug reactions: primetime and not ready for primetime tests. J Allergy Clin Immunol. 2016;138(4): 943-955. doi:10.1016/j.jaci.2016.08.002. Erratum in: J Allergy Clin Immunol 2017;139(1):378

5. Kimpton JE, Carey IM, Threapleton CJD, et al. Longitudinal exposure of English primary care patients to pharmacogenomic drugs: an analysis to inform design of pre-emptive pharmacogenomic testing. $\mathrm{Br}$ J Clin Pharmacol. 2019;85(12):2734-2746. doi:10.1111/bcp.14100

6. Pirmohamed M, James S, Meakin S, et al. Adverse drug reactions as cause of admission to hospital: prospective analysis of 18820 patients. BMJ. 2004;329(7456):15-19. doi:10.1136/bmj.329.7456.15

7. Sukasem C, Chantratita W. A success story in pharmacogenomics: genetic ID card for SJS/TEN. Pharmacogenomics. 2016;17(5):455-458. doi:10.2217/pgs-2015-0009

8. Blagec $K$, Koopmann $R$, Crommentuijn-van Rhenen $M$, et al. Implementing pharmacogenomics decision support across seven European countries: the Ubiquitous Pharmacogenomics (U-PGX) project. J Am Med Inform Assoc. 2018;25(7):893-898. doi: 10.1093/jamia/ocy005

9. Raza S, Blackburn L, Moorthie S, Cook S, Johnson E, Gaynor L. The personalised medicine technology landscape. Cambridge: PHG Foundation; 2018.

10. Smith LB, Golberstein E, Anderson K, et al. The association of EHR drug safety alerts and co-prescribing of opioids and benzodiazepines. J Gen Intern Med. 2019;34(8):1403-1405. doi:10.1007/s11606-01904985-w

11. Paterno MD, Maviglia SM, Gorman PN, et al. Tiering drug-drug interaction alerts by severity increases compliance rates. J Am Med Inform Assoc. 2009;16(1):40-46. doi:10.1197/jamia.M2808

12. Relling MV, Evans WE. Pharmacogenomics in the clinic. Nature. 2015; 526(7573):343-350. doi:10.1038/nature15817

13. Appelbaum PS, Parens E, Berger SM, Chung WK, Burke W. Is there a duty to reinterpret genetic data? The ethical dimensions. Genet Med. 2020;22(3):633-639. doi:10.1038/s41436-019-0679-7

14. Dolin RH, Boxwala A, Shalaby J. A pharmacogenomics clinical decision support service based on FHIR and CDS hooks. Methods Inf Med. 2018;57(SO2):e115-e123. doi:10.1055/s-0038-1676466

15. NHS England. Clinical Commissioning Urgent Policy Statement: Pharmacogenomic testing for DPYD polymorphisms with fluoropyrimidine therapies. [Internet]. 2020. https://www.england.nhs. uk/wp-content/uploads/2020/11/1869-dpyd-policy-statement.pdf. Accessed June 20, 2021

16. Peterson JF, Field JR, Shi Y, et al. Attitudes of clinicians following large-scale pharmacogenomics implementation. Pharmacogenomics $\mathrm{J}$. 2016;16(4):393-398. doi:10.1038/tpj.2015.57

17. Veilleux S, Bouffard M, Bourque BM. Patient and health care provider needs and preferences in understanding pharmacogenomic and genomic testing: a meta-data analysis. Qual Health Res. 2020;30(1):43-59. doi:10.1177/1049732319858325

18. The PRSB. Guidance for using pharmacogenomic information in clinical practice. [Internet]. 2020. https://theprsb.org/wp-content/ uploads/2020/11/Pharmacogenomics-Report_V1.0.pdf. Accessed June 20, 2021.

19. Wake DT, Smith DM, Kazi S, Dunnenberger HM. Pharmacogenomic clinical decision support: a review, how-to guide, and future vision. Clin Pharmacol Ther. 2021. doi:10.1002/cpt.2387. Epub ahead of print.

\section{SUPPORTING INFORMATION}

Additional supporting information may be found in the online version of the article at the publisher's website.

How to cite this article: Carter J-PL, Critchlow J, Jackson S, et al. Pharmacogenomic alerts: Developing guidance for use by healthcare professionals. Br J Clin Pharmacol. 2022;1-10. doi:10.1111/bcp.15234 